\title{
Increase in the Random Dopant Induced Threshold Fluctuations and Lowering in Sub-100 nm MOSFETs Due to Quantum Effects: A 3-D Density-Gradient Simulation Study
}

\author{
Asen Asenov, Member, IEEE, Gabriela Slavcheva, Andrew R. Brown, John H. Davies, and Subhash Saini
}

\begin{abstract}
In this paper, we present a detailed simulation study of the influence of quantum mechanical effects in the inversion layer on random dopant induced threshold voltage fluctuations and lowering in sub-100 $\mathrm{nm}$ MOSFETs. The simulations have been performed using a three-dimensional (3-D) implementation of the density gradient (DG) formalism incorporated in our established 3-D atomistic simulation approach. This results in a self-consistent 3-D quantum mechanical picture, which implies not only the vertical inversion layer quantization but also the lateral confinement effects related to current filamentation in the "valleys" of the random potential fluctuations. We have shown that the net result of including quantum mechanical effects, while considering statistical dopant fluctuations, is an increase in both threshold voltage fluctuations and lowering. At the same time, the random dopant induced threshold voltage lowering partially compensates for the quantum mechanical threshold voltage shift in aggressively scaled MOSFETs with ultrathin gate oxides.
\end{abstract}

Index Terms-Dopant fluctuation, quantum effects, MOSFETs, numerical simulation, 3-D, threshold.

\section{INTRODUCTION}

$\mathbf{M}$ OSFET threshold voltage variation due to statistical fluctuations in the number and position of dopant atoms [1]-[4] becomes a serious problem when MOSFETs are scaled to sub-100 nm dimensions [5]-[8]. This is complemented by a pronounced threshold voltage lowering [4], [8] associated with current percolation through valleys in the potential distribution at the interface due to the random position of dopants. At the same time the increase in doping concentration to above $1 \times 10^{18} \mathrm{~cm}^{-3}$, and the reduction in the oxide thickness to below $3 \mathrm{~nm}$ in sub-100 nm MOSFETs [5], result in a large surface electric field, even near threshold, and strong quantization in the direction perpendicular to the channel [9]-[11], with a corresponding increase in threshold voltage, and reduction in gate capacitance and drive [12]-[15].

The realistic modeling of dopant fluctuation effects in deep submicron MOSFETs requires a three-dimensional (3-D) nu-

Manuscript received August 11, 2000. This work was supported by NASA Ames Research Center Grant NAG 2-1241. The review of this paper was arranged by Editor G. Baccarani.

A. Asenov, G. Slavcheva, A. R. Brown, and J. H. Davies are with the Device Modeling Group, Department of Electronics and Electrical Engineering, University of Glasgow, Glasgow G12 8QQ, U.K.

S. Saini is with the NASA Ames Research Center, Moffett Field, CA 94035 USA.

Publisher Item Identifier S 0018-9383(01)02351-6. merical simulation, with fine grain discretization, and a statistical analysis of the results from the simulation of a statistical sample of devices [4], [8], [16]. All previous 3-D simulation studies of random dopant fluctuation effects [4], [7], [8], [16]-[21] use a simple drift-diffusion or, in one case, hydrodynamic [22] approximation, and, with the exception of [22], do not take into account quantum effects. However, [22] does not explicitly demonstrate the role of the quantum effects on MOSFET parameter fluctuations. Until now, it was unclear to what extent the quantum effects would affect the random dopant induced threshold voltage fluctuations and lowering in aggressively scaled MOSFETs with ultrathin gate oxides, and to what degree the threshold voltage lowering may compensate for the increase in the threshold voltage associated with inversion layer quantization.

In this paper, we study the influence of the quantum effects in the inversion layer on the random dopant induced threshold voltage fluctuations and lowering in sub-100 nm MOSFETs. The quantum mechanical effects are incorporated in our established 3-D atomistic simulation approach [8], [16] using a 3-D implementation of the density gradient (DG) model [23]. This results in a self-consistent, fully 3-D quantum mechanical picture which accounts for the vertical inversion layer quantization, lateral confinement effects associated with the current filamentation in the "valleys" of the potential fluctuation and, according to [24], [25], tunneling through the sharp potential barriers associated with individual dopants. The next section describes the 3-D implementation of the DG model in an atomistic context, outlining the equations, the solution domain, the boundary conditions and the numerical procedures. In Section III, we calibrate and validate the DG simulations with respect to comprehensive quantum mechanical calculations based on the full band formalism [11], comparing the published quantum mechanical threshold voltage shifts and inversion layer charge distributions with the results of DG simulations. Three-dimensional atomistic simulation results highlighting the influence of the quantum mechanical effects on the random dopant induced threshold voltage fluctuations and lowering are presented and analyzed in Section IV.

\section{SIMULATION APPROACH}

A hydrodynamic interpretation of the quantum mechanics and quantum corrections to the fluid equations was proposed 
in the late 1920s [26], and elaborated further by several authors [27]. In a form similar to the form adopted in this paper, DG quantum corrections have been used in two-dimensional (2-D) hydrodynamic simulations of MESFETs and HEMTs [28]. As demonstrated in [29], to lowest order, the quantum system behaves as an ideal gradient gas for typical low-density and hightemperature semiconductor conditions. Assuming a scalar effective mass, quantum corrections have been included in the drift-diffusion set of semiconductor equations by introducing an additional term in the carrier flux expression

$$
F_{n}=n \mu_{n} \nabla \psi-D_{n} \nabla n+2 \mu_{n} \nabla\left(b_{n} \frac{\nabla^{2} \sqrt{n}}{\sqrt{n}}\right)
$$

where $b_{n}=\hbar^{2} /\left(12 q m_{n}^{*}\right)$ and all other symbols have the conventional meaning. The quantum correction term in (1) is referred to as "quantum diffusion" since its inclusion yields a theory that contains both quantum confinement effects and quantum mechanical tunneling [24], [25], [29]. A robust approach has been proposed in [23] to avoid the discretization of fourth-order derivatives when using (1) in multidimensional numerical simulations. By introducing a generalized electron quasi-Fermi potential $\phi_{n}$ according to the expression $F_{n}=n \mu_{n} \nabla \phi_{n}$, the unipolar drift-diffusion system of equations with QM corrections, which in many cases is sufficient for MOSFET simulations, becomes

$$
\begin{aligned}
\nabla \cdot(\varepsilon \nabla \psi) & =-q\left(p-n+N_{D}^{+}-N_{A}^{-}\right) \\
2 b_{n} \frac{\nabla^{2} \sqrt{n}}{\sqrt{n}} & =\phi_{n}-\psi+\frac{k T}{q} \ln \frac{n}{n_{i}} \\
\nabla \cdot\left(n \mu_{n} \nabla \phi_{n}\right) & =0
\end{aligned}
$$

where $\psi, \phi_{n}$, and $\sqrt{n}$ are independent variables. The right-hand side of (3) represents the Boltzmann statistics for electrons and the left-hand side can be interpreted as a quantum mechanical correction to the Boltzmann statistics. At the same time, (3) is a nonlinear partial differential equation, which closely resembles the Schrödinger equation, and a microscopic expression for the macroscopic factor $b_{n}$ has been derived in [29] based on this analogy.

Similar to the approach outlined in [16], at low drain voltage, in linear mode of MOSFET operation, we consider a constant quasi-Fermi potential in the simulations which decouples (2) and (3) from (4). Therefore, we solve self-consistently the 3-D Poisson equation (2) for the potential, and (3) for the electron concentration. In the iterative solution process the electron concentration obtained from the solution of (3), together with Boltzmann statistics for the hole concentration $p$, are used in the solution of (2).

A typical atomistic simulation domain used in the simulation of a $30 \times 50 \mathrm{~nm}$ n-channel MOSFET with oxide thickness $t_{\mathrm{ox}}=3 \mathrm{~nm}$ and a junction depth $x_{j}=7 \mathrm{~nm}$ is outlined in Fig. 1. The uniform doping concentration in the channel region $N_{A}=5 \times 10^{18} \mathrm{~cm}^{-3}$ is resolved down to an individual dopant level using fine grain discretization with typical mesh spacing $0.5 \mathrm{~nm}$, much less than the typical spacing between impurities. The number of dopants in the random dopant region of each individual transistor follows a Poisson distribution. The position of dopants is chosen at random and each dopant is assigned to the nearest grid node. By varying the mesh spacing we have checked that the error associated with the charge assignment is less than $1 \%$ for the devices simulated in this paper. More complex doping profiles in the random dopant region of the device may be introduced using a rejection technique.

Standard boundary conditions are used for the potential in the Poisson equation (2) with zero bias applied at the source and drain contacts according to the adopted constant quasi-Fermi level approximation. Dirichlet boundary conditions are applied to electrons in the DG equation (3) at the contacts and $\mathrm{Si} / \mathrm{SiO}_{2}$ interface introducing charge neutrality and vanishingly small values, respectively, and Neumann boundary conditions are applied at all other boundaries of the solution domain. One step Newton-SOR iterations are used for solving both the Poisson equation (2) and the DG equation (3) [30]. At the beginning of the self-consistent iteration, the nonlinear Poisson equation is solved using Boltzmann statistics for both electrons and holes.

The current at low drain voltage is calculated by solving a simplified current continuity equation (5), in a drift approximation only [16], in a thin slab near the $\mathrm{Si} / \mathrm{Si}_{2}$ interface engulfing the inversion layer charge

$$
\nabla \cdot \mu_{n} n \nabla V=0
$$

Dirichlet boundary conditions are applied for the "driving" potential $V$ at the source and drain contacts, with $V=0$ and $V=$ $V_{D}$, respectively, and Neumann boundary conditions are applied at all other boundaries of the slab. Drain voltage $V_{D}=10 \mathrm{mV}$ is used in all simulations. The current is extracted by integrating the drift current density $J_{n}=\mu_{n} n \nabla V$ along a cross section of the slab. The described procedure is equivalent to calculating the conductance of the device. We have demonstrated [16] that, at low drain voltage, in the drift-diffusion approximation, the approach described above reproduces with high accuracy the results obtained from the self consistent solution of the Poisson and the electron drift-diffusion equation.

The current criterion $I_{T}=10^{-8} W_{\text {eff }} / L_{\text {eff }}$ [A] is used to estimate the threshold voltage $V_{T}$. Typically, samples of $200 \mathrm{mi}-$ croscopically different transistors are simulated for each combination of macroscopic design parameters, in order to extract the average threshold voltage $\left\langle V_{T}\right\rangle$, and its standard deviation $\sigma V_{T}$. The corresponding relative standard deviation of the extracted $\sigma V_{T}$ is $\sigma_{\sigma V_{T}}=5 \%$ for all results presented in this paper.

Fig. 2 illustrates the potential distribution obtained from the self-consistent solution of (2) and (3) in the solution domain outlined in Fig. 1 at gate voltage equal to the threshold voltage. Strong potential fluctuations at the $\mathrm{Si} / \mathrm{SiO}_{2}$ interface associated with the discrete dopants can be observed. One electron equiconcentration contour which corresponds to this solution is presented in Fig. 3. The equi-concentration contour highlights the basic features of the quantum charge distribution. The quantum confinement in the channel results in a maximum in the electron concentration which is located approximately $1.5 \mathrm{~nm}$ below the interface. The 3-D solution of (3), within the limitations of the scalar effective mass approximation, captures the lateral confinement in current channels percolating through the "valleys" in the fluctuation surface potential. 


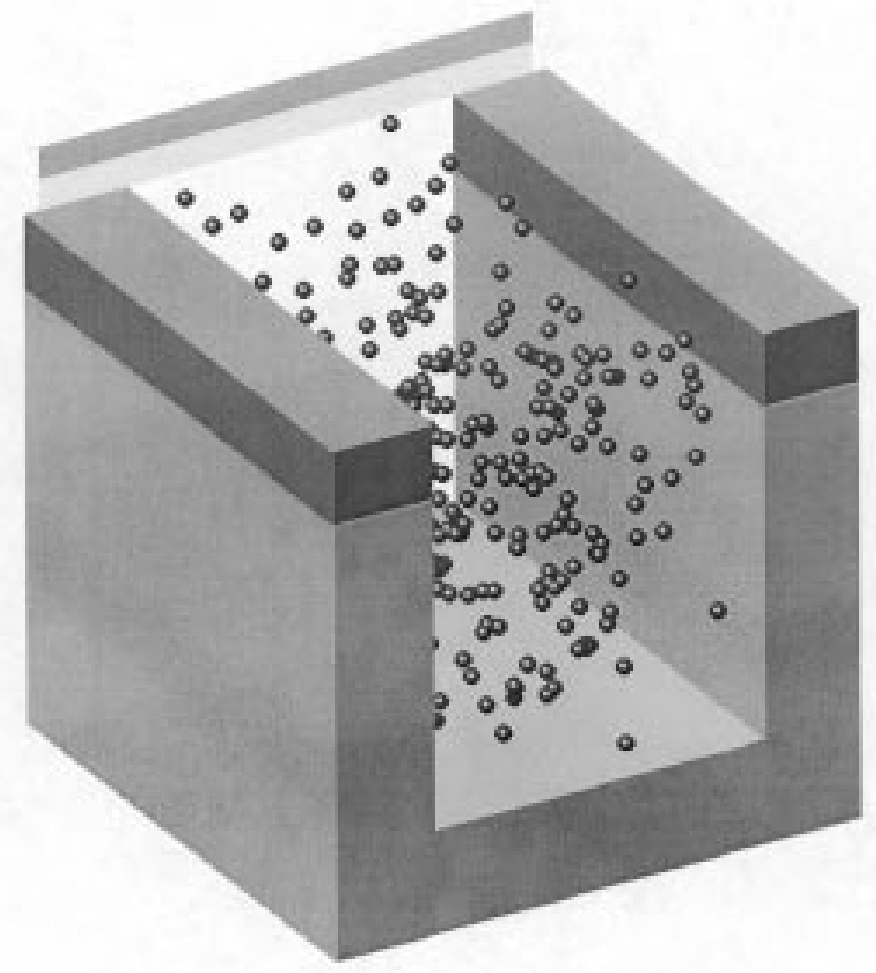

Fig. 1. Typical atomistic simulation domain and dopant distribution used in the simulation of a $30 \times 50 \mathrm{~nm}$ n-channel MOSFET with oxide thickness $t_{\mathrm{ox}}=3$ $\mathrm{nm}$, junction depth $x_{j}=7 \mathrm{~nm}$, and channel acceptor concentration $N_{A}=$ $5 \times 10^{18} \mathrm{~cm}^{-3}$.

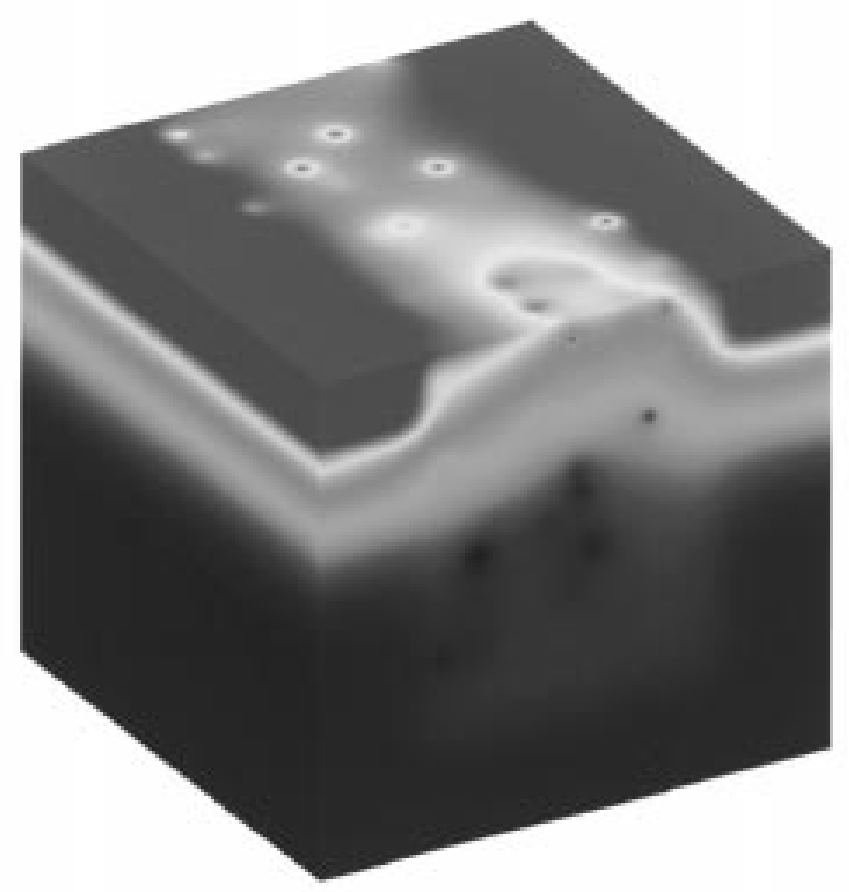

Fig. 2. Potential distribution at threshold voltage obtained from the atomistic DG simulation of a $30 \times 50 \mathrm{~nm}$ MOSFET with design parameters given in Fig. 1.

\section{CALIBRATION}

Since the DG model accounts for lowest order quantum effects, and (3) can be considered only as an approximation to the

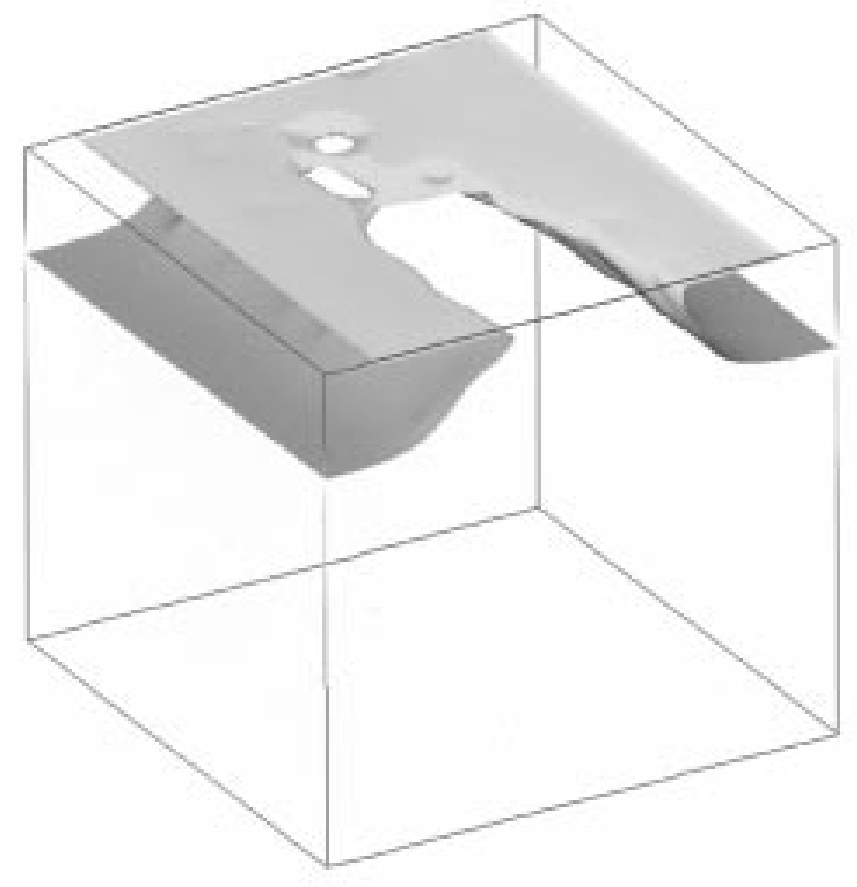

Fig. 3. One equi-concentration contour corresponding to the potential distribution in Fig. 2. The inversion charge distribution peaks below the $\mathrm{Si} / \mathrm{SiO}_{2}$ interface.

Schrödinger equation, the DG model has to be carefully validated (and eventually calibrated) against a full, self-consistent solution of the Poisson-Schrödinger equation before being used further in our atomistic simulations. To some extent such validation/calibration has already been carried out in [23] in comparison with an ellipsoidal band Poisson-Schrödinger solver, but only in respect of $C-V$ calculations. Here, we are more concerned about correct prediction of the threshold voltage shift and the quantum mechanical charge distribution in the inversion layer, which will be affected further by the random dopant induced potential fluctuations.

The DG validation/calibration is a difficult task in 3-D, particularly in a complex solution domain representing a MOSFET, and potential incorporating fluctuations from discrete dopants. Therefore we validate the DG approach against rigorous full band Poisson-Schrödinger simulations [11] in the one-dimensional (1-D) case only, and for continuous doping. The DG results for the quantum mechanical threshold voltage shift $V_{T}(Q M)-V_{T}($ Classical $)$, shown in Fig. 4, using a value of electron effective mass $m^{*}=0.19 m_{0}$, as recommended in [23], are in good agreement with the shift reported in [11]. The range of doping concentrations in this comparison corresponds to the channel doping in properly scaled MOSFETs with channel lengths below $100 \mathrm{~nm}$. Although the above value of the effective mass is identical to the transverse electron mass in $\mathrm{Si}$, there is no physical reason for using the transverse electron mass in (3). Indeed in the [001] direction considered here, the interface confinement lifts the degeneracy of the sixfold ellipsoidal (at low $k$ ) conducting bands of Si. The total electron concentration in the inversion layer is composed of the electron concentrations in the subbands corresponding to the two ellipsoids with longitudinal effective mass normal to the interface 


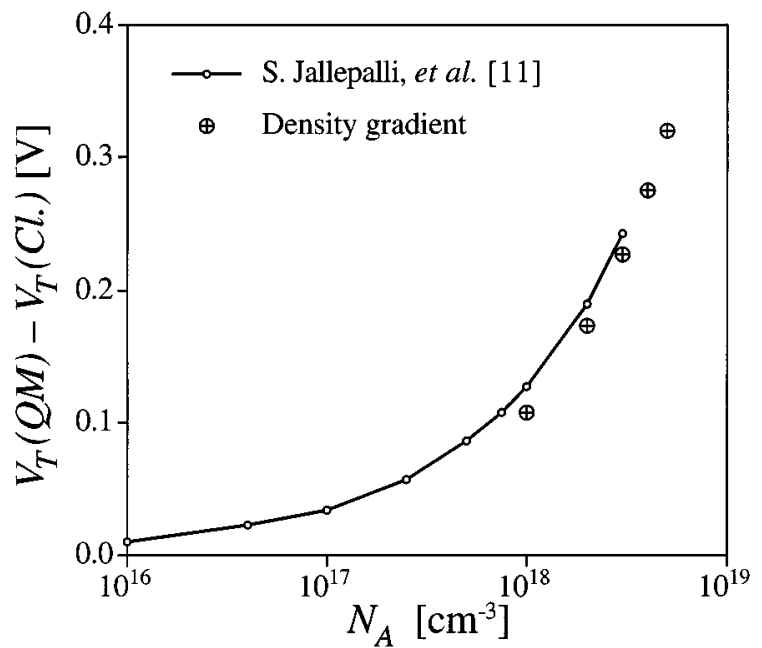

Fig. 4. Quantum mechanical threshold voltage shift as a function of the doping concentration. A comparison between 1-D DG and full band Poisson-Schrödinger results [11] at oxide thickness $t_{\mathrm{ox}}=4 \mathrm{~nm}$.

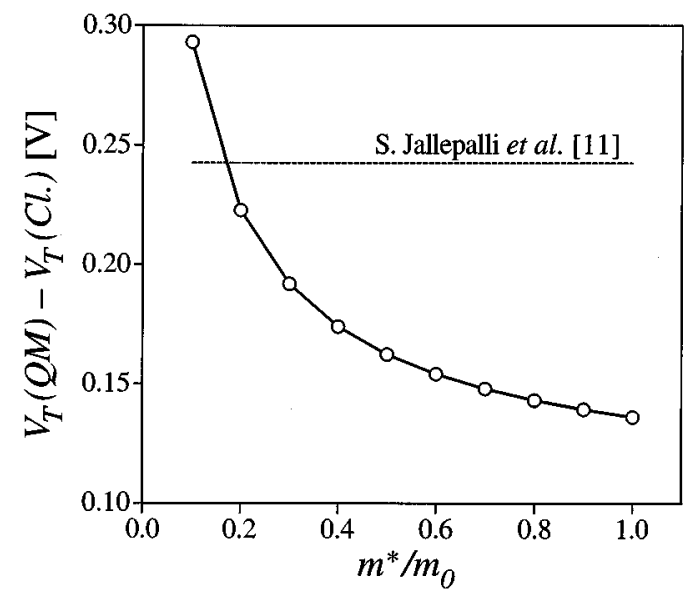

Fig. 5. Quantum mechanical threshold voltage shift as a function of the electron effective mass $m^{*}$, in 1-D DG simulation for acceptor concentration $N_{A}=3 \times 10^{18} \mathrm{~cm}^{-3}$, and oxide thickness $t_{\mathrm{ox}}=4 \mathrm{~nm}$.

$\left(m^{*}=0.916 m_{0}\right)$ and the four ellipsoids with transfer effective mass parallel to the interface $\left(m^{*}=0.19 m_{0}\right)$. Although not only the lowest subband related to $m^{*}=0.916 m_{0}$ is occupied at room temperature and contributions from the subbands corresponding to $m^{*}=0.19 m_{0}$ can be expected, the effective mass needed to calibrate the DG model is unphysically low and has to be treated as a fitting parameter. To illustrate this point further, we present in Fig. 5 the dependence of the quantum mechanical threshold voltage shift as a function of the value of $m^{*}$ used in the DG simulations for doping concentration $N_{A}=3 \times 10^{18} \mathrm{~cm}^{-3}$ and oxide thickness $t_{\mathrm{ox}}=4 \mathrm{~nm}$. Exact agreement with the shift predicted in [11] at this doping concentration occurs for $m^{*}=0.175 m_{0}$ which is even lower that the value suggested in [23].

Fig. 6 compares the electron concentration distributions obtained using the DG model with a full band Poisson-Schrödinger simulation. The parameters in both simulations are selected to allow a direct comparison with the results presented in [11]. Good agreement between the electron

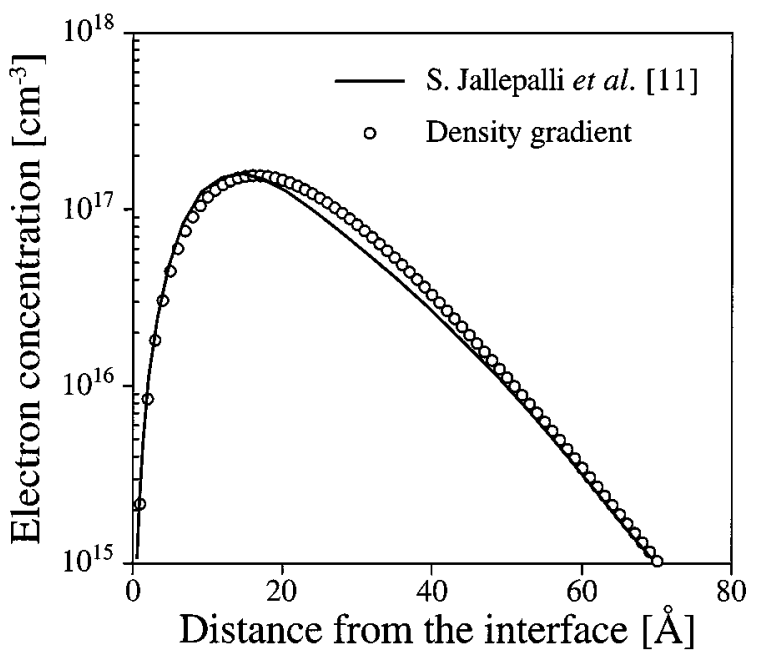

Fig. 6. Comparison of the 1-D charge distribution obtained from DG and full band Poisson-Schrödinger simulations for acceptor concentration $N_{A}=5 \times$ $10^{17} \mathrm{~cm}^{-3}$, oxide thickness $t_{\mathrm{ox}}=4 \mathrm{~nm}$, and inversion charge density $1.67 \times$ $10^{11} \mathrm{~cm}^{-2}$.

distributions obtained from the two models is observed with an inversion charge distribution slightly narrower and a charge centroid slightly closer to the interface in the DG simulation. Similar level of accuracy has to be expected in the [010] and the [100] directions which are equivalent to the [001] direction in terms of band structure and the expected quantum confinement effects. In an arbitrary direction we have to accept the limitation of the single effective mass in this first attempt to incorporate quantum mechanical effects in the 3-D "atomistic" simulation picture.

Although theoretically the DG approach also accounts for tunneling [24], [25], which in our case may include the tunneling through the Coulomb potential of individual dopants, no attempt has been made to calibrate the DG approach in respect of this phenomenon.

\section{RESUlTS AND DISCUSSION}

In this section, we compare the results of DG atomistic simulations with previously published, purely classical atomistic simulation results [8], in order to highlight the influence of the quantum effects on the random dopant induced threshold voltage fluctuations and lowering.

The dependence of the threshold voltage on oxide thickness, obtained from classical and from quantum DG simulations, is presented in Fig. 7 for MOSFETs with uniform doping concentration in the channel region $N_{A}=5 \times 10^{18} \mathrm{~cm}^{-3}$, effective channel length $L_{\text {eff }}=50 \mathrm{~nm}$, channel width $W_{\text {eff }}=$ $50 \mathrm{~nm}$, and junction depth $x_{j}=7 \mathrm{~nm}$. Results for the average threshold voltage $\left\langle V_{T}\right\rangle$ obtained from atomistic simulations, and for the threshold voltage $V_{T 0}$ obtained from continuous charge simulations are compared. For completeness we provide here and in all following figures also results which take into account the poly-Si depletion and the random dopant distribution in the poly-Si gate, together with the quantum effects, in a manner described in detail in [31]. The poly-Si doping concentration used throughout the paper is $N_{D}=1 \times 10^{20}$ 


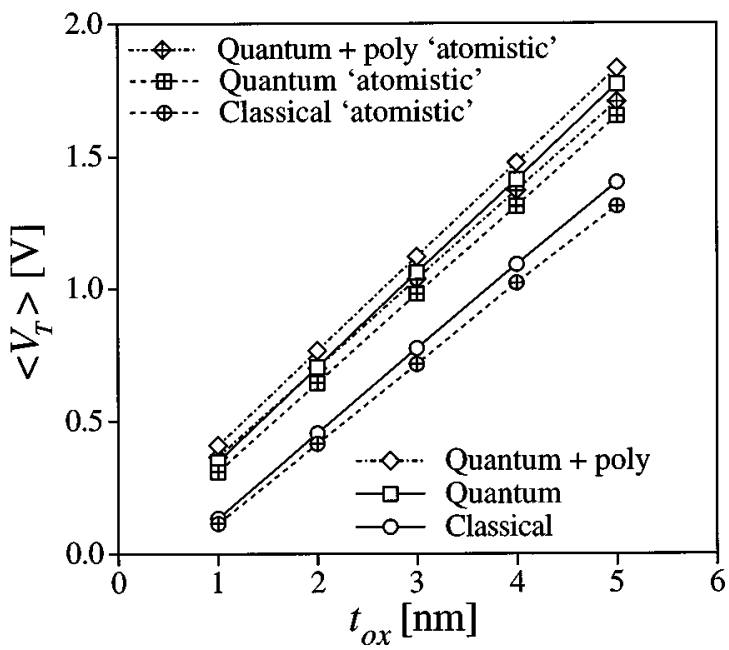

Fig. 7. Threshold voltage as a function of the oxide thickness in $50 \times 50 \mathrm{~nm}$ MOSFETs with channel doping concentration $N_{A}=5 \times 10^{18} \mathrm{~cm}^{-3}$ and poly-Si gate doping $N_{D}=1 \times 10^{20} \mathrm{~cm}^{-3}$.

$\mathrm{cm}^{-3}$. It can be noted that for oxide thickness $t_{\mathrm{ox}}=4 \mathrm{~nm}$ the quantum mechanical shift in the threshold voltage obtained from full 3-D DG simulation of MOSFETs with continuous doping agree well with the 1-D DG result presented in Fig. 4. As expected [11], the quantum mechanical threshold voltage shift decreases with the reduction in the oxide thickness. The inclusion of the poly-Si gate in the simulations results in additional increase in the threshold voltage. Most importantly, the random dopant induced threshold voltage lowering, inherent to the atomistic simulations, and associated with percolation of the channel current through "valleys" in the potential fluctuations, is enhanced in the quantum case. This becomes clear from Fig. 8 where the threshold voltage lowering $\left\langle V_{T}\right\rangle-V_{T 0}$ extracted from the data presented in Fig. 7 is plotted as a function of the oxide thickness and compared for the classical and quantum simulations. Although the magnitude of the threshold voltage lowering decreases almost linearly with the reduction of the oxide thickness in both cases, the relative quantum mechanical increase of the lowering becomes larger than $50 \%$ for oxide thicknesses below $2 \mathrm{~nm}$ and reaches almost 100\% for the limiting oxide thickness of $1 \mathrm{~nm}$.

The dependencies of the threshold voltage standard deviation $\sigma V_{T}$ as a function of the oxide thickness, extracted from classical and from quantum atomistic simulations, are compared in Fig. 9 for MOSFETs with the same parameters as the devices in Fig. 7. In the classical simulations $\sigma V_{T}$ scales linearly to zero with the corresponding scaling of $t_{\mathrm{ox}}$, within the accuracy of the statistical estimations. This derives from the fact that a Dirichlet boundary condition was applied at the gate electrode during the simulations, keeping constant the value of the potential on top of the gate insulator. Such a "metal gate" boundary condition completely screens and flattens the potential fluctuations at the $\mathrm{Si} / \mathrm{SiO}_{2}$ interface when the oxide becomes infinitesimally thin. This in turns kills the threshold voltage fluctuations since the maximum of the classical inversion layer charge distribution and channel current density occurs at the interface. The values of $\sigma V_{T}$ corresponding to the quantum simulations are shifted up with respect to the classical simulations, and the shift increases

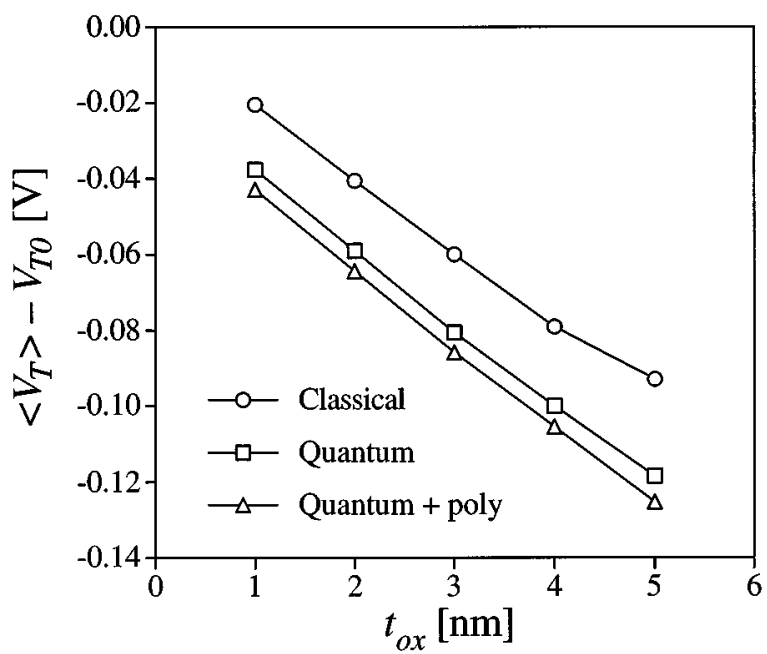

Fig. 8. Threshold voltage lowering as a function of the oxide thickness extracted from the data in Fig. 7.

slightly with the increase in the oxide thickness. This shift can be partially explained by the fact that the inversion layer centroid in the quantum simulations is below the interface and results in an increase in the effective oxide thickness. Using a relatively crude approximation, this can be taken into account simply by shifting the straight line approximating the classical dependence of $\sigma V_{T}$ on the oxide thickness along the oxide thickness axis by

$$
\Delta=\varepsilon_{\mathrm{ox}} z_{i} / \varepsilon_{\mathrm{Si}}
$$

where $z_{i}$ is the inversion layer centroid. According to [32], at doping concentration $N_{A}=5 \times 10^{18} \mathrm{~cm}^{-3}$ the inversion layer centroid is $z_{i}=1.12 \mathrm{~nm}$ which corresponds to a shift of $\Delta=$ $0.37 \mathrm{~nm}$. The classical $\sigma V_{T}$ dependence shifted by this value lies below but rather close to the values of $\sigma V_{T}$ obtained from the quantum mechanical. This is an indication that a substantial fraction of the quantum increase of the threshold voltage fluctuations can be attributed to the effective increase in the oxide thickness. Other factors like the lateral confinement effects in the current channels and the quantum mechanical broadening of the inversion layer charge have additional contributions.

It should be noted also that the inclusion of the poly-Si gate in the simulations results in additional increase of $\sigma V_{T}$ which, in combination with the increase associated with the quantum mechanical effects, almost doubles the threshold voltage fluctuations for oxide thicknesses below $2 \mathrm{~nm}$.

The atomistically simulated average threshold voltage $\left\langle V_{T}\right\rangle$ for a set of MOSFETs with different channel lengths is compared in Fig. 10 with the threshold voltage $V_{T 0}$ of devices with continuous doping. The devices have oxide thickness $t_{\mathrm{Ox}}=$ $3 \mathrm{~nm}$, and all other parameters similar to the MOSFETs from Fig. 7. Both results from classical atomistic simulation and simulations including DG correction for the quantum mechanical effects are presented in the same figure. Let us focus first on the classical and the quantum mechanical simulations with continuous doping. The quantum mechanical shift in the threshold voltage exhibits a channel length dependence, and decreases with the reduction in the channel length, from $292 \mathrm{mV}$ at $L_{\mathrm{eff}}=$ $100 \mathrm{~nm}$ to $271 \mathrm{mV}$ at $L_{\text {eff }}=30 \mathrm{~nm}$. This can be interpreted as an 


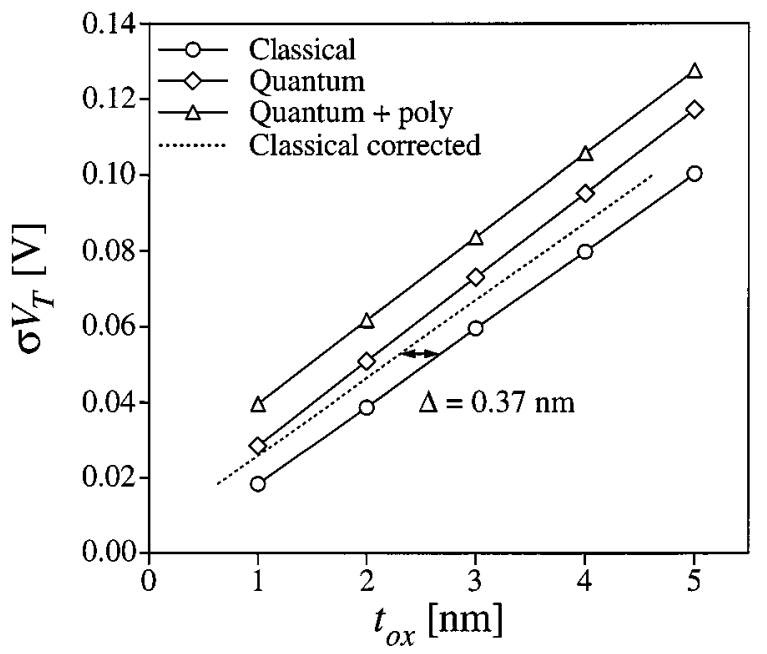

Fig. 9. Threshold voltage standard deviation as a function of the oxide thickness for devices with the same parameters as in Fig. 7.

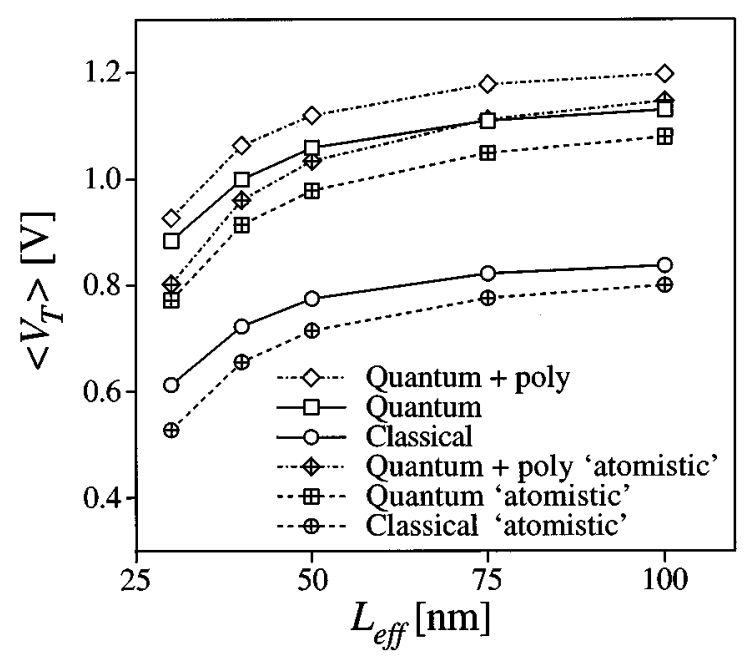

Fig. 10. Threshold voltage as a function of the effective gate length for MOSFETs with channel width $W_{\text {cff }}=50 \mathrm{~nm}$, channel doping concentration $N_{A}=5 \times 10^{18} \mathrm{~cm}^{-3}$, oxide thickness $t_{\mathrm{ox}}=3 \mathrm{~nm}$, and poly-Si gate doping $N_{D}=1 \times 10^{20} \mathrm{~cm}^{-3}$.

increase in the short-channel effects in the quantum mechanical simulations as a result of an increase in effective oxide thickness associated with the location of the inversion charge centroid below the $\mathrm{Si} / \mathrm{Si}_{2}$ interface. The inclusion of the poly-Si gate increases even more the effective oxide thickness as a result of the poly-depletion and results in a further increase in the short-channel effects.

In order to interpret clearly the results of the atomistic simulations presented in Fig. 10 we compare in Fig. 11 the corresponding threshold voltage lowering, $\left\langle V_{T}\right\rangle-V_{T 0}$, in the classical and quantum case. The threshold voltage lowering in the quantum mechanical atomistic simulations increases faster than the threshold voltage lowering in the classical simulations with the reduction of the channel length. This can be interpreted as an additional increase of the short-channel effects in the quantum mechanical atomistic case. Bearing in mind that the threshold voltage lowering results from an early percolation of

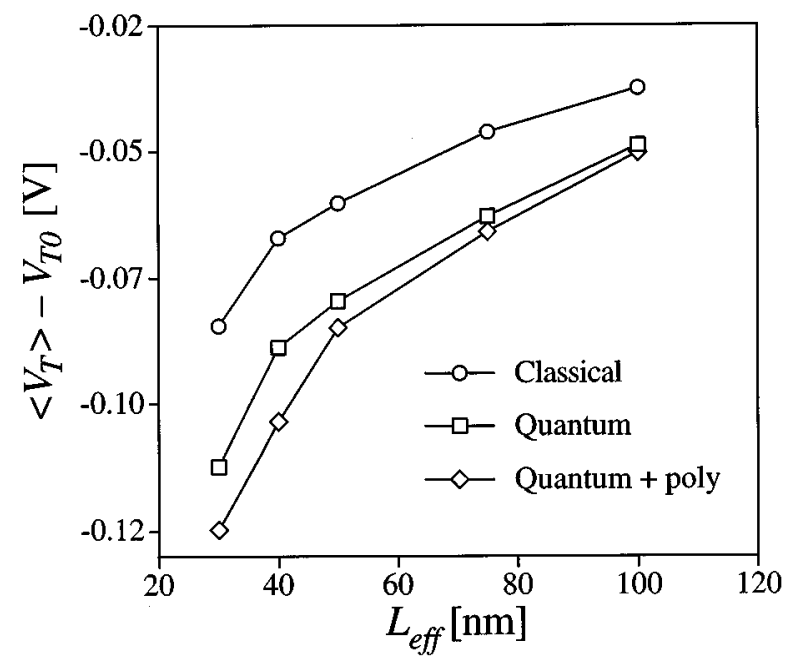

Fig. 11. Threshold voltage lowering as a function of the effective channel length extracted from the data in Fig. 10.

current through valleys in the potential fluctuations in the plane of current flow we speculate that the increase of the threshold voltage lowering with the reduction of the channel length has two aspects. First the length of the percolation paths decreases which reduces the percolation threshold. Secondly the discrete doping distribution results in localized regions with higher and lower than the average doping concentrations. In the regions with lower doping concentration the 2-D effects associated with the penetration of the source/drain potential in the channel are stronger and in interaction with dominant percolation paths this results in further threshold voltage lowering when the channel length is reduced. In the quantum mechanical case the increased effective thickness of the oxide increases further the influence of the source/drain potential on the potential distribution and lowering in the channel region and hence through the second mechanism increases the threshold voltage lowering. The threshold voltage lowering, which reaches more than $110 \mathrm{mV}$ in a 30-nm MOSFET, compensates for a significant portion of the quantum mechanical threshold voltage shift.

Finally, Fig. 12 compares channel length dependence of the standard deviations in the threshold voltage $\sigma V_{T}$ calculated using classical and quantum mechanical atomistic simulations. The quantum mechanical increase in $\sigma V_{T}$ is more pronounced at the shorter channel lengths and ranges from $23 \%$ at the 100 $\mathrm{nm}$ MOSFETs to $25 \%$ at transistors with $30 \mathrm{~nm}$ channel length. The inclusion of the poly-Si gate in the simulations increases $\sigma V_{T}$ by another $15 \%$ over the whole range of channel lengths.

\section{CONCLUSIONS}

In this paper, we have presented a 3-D atomistic DG simulation approach for determining the threshold voltage in aggressively scaled MOSFETs, which takes into account both the discrete random dopant distribution in the channel region and the quantum effects in the inversion layer. It accounts for random dopant induced threshold voltage fluctuations and lowering, and quantum mechanical threshold voltage shift in a self consistent manner. 


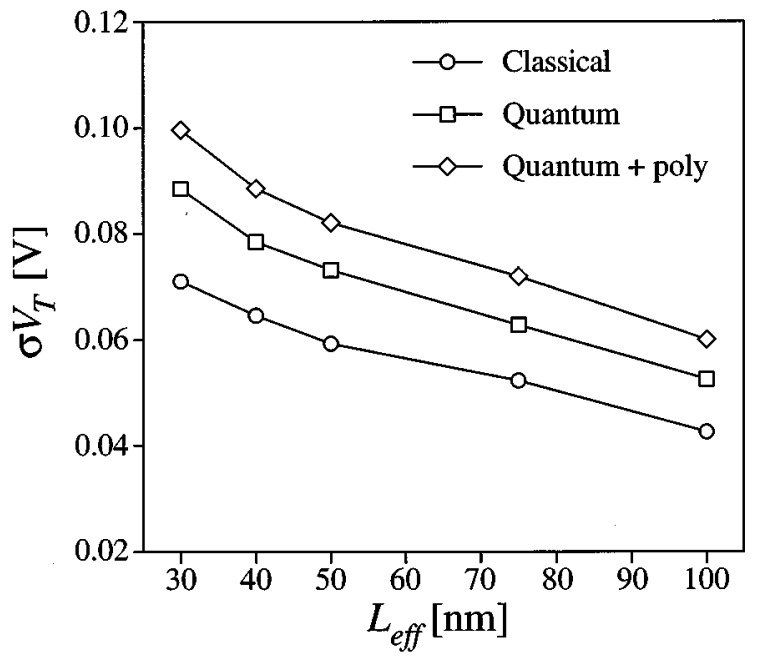

Fig. 12. Threshold voltage standard deviation as a function of the effective channel length for devices with channel doping concentration $N_{A}=5 \times 10^{18}$ $\mathrm{cm}^{-3}$ and poly-Si gate doping $N_{D}=1 \times 10^{20} \mathrm{~cm}^{-3}$.

We have demonstrated that the introduction of quantum effects in the previously published 3-D statistical atomistic simulations results in an increase in both threshold voltage fluctuations and lowering. The quantum increase in the threshold voltage fluctuations amounts to more than 50\% in MOSFETs with oxide thicknesses below $1.5 \mathrm{~nm}$, expected near the end of the Silicon Roadmap [5]. If, in addition, the poly-Si depletion and the discrete random dopants in the poly-Si gate are taken into account, the increase reaches $100 \%$ in devices with ultrathin gate oxides. At the same time, the quantum mechanical increase in the threshold voltage becomes partially compensated by the threshold voltage lowering due to atomistic effects. This compensation varies from $16 \%$ in $100 \mathrm{~nm}$ MOSFETs up to $40 \%$ in 30-nm devices.

The combination of quantum mechanical and random dopant effects, which are closely interlinked and may enhance or compensate each other, becomes very important in sub-100 nm MOSFETs and poses serious challenges for the development of the next generation of simulation tools.

\section{REFERENCES}

[1] B. Hoeneisen and C. A. Mead, "Fundamental limitations in microelectronics-I, MOS technology," Solid-State Electron., vol. 15, pp. 819-829, 1972.

[2] R. W. Keyes, "Physical limits in digital electronics," Proc. IEEE, vol. 63, pp. 740-766, 1975.

[3] T. Mizuno, J. Okamura, and A. Toriumi, "Experimental study of threshold voltage fluctuation due to statistical variation of channel dopant number in MOSFET's," IEEE Trans. Electron Devices, vol. 41, pp. 2216-2221, 1994.

[4] H.-S. Wong and Y. Taur, "Three dimensional 'atomistic' simulation of discrete random dopant distribution effects in sub-0.1 $\mu \mathrm{m}$ MOSFETs," in IEDM Tech. Dig., 1993, pp. 705-708.

[5] The National Technology Road-map for Semiconductors. San Jose, CA: Semiconductor Industry Assoc., 1997.

[6] J. T. Horstmann, U. Hileringmann, and K. F. Gosser, "Matching analysis of deposition defined 50-nm MOSFET's," IEEE Trans. Electron Devices, vol. 45, pp. 299-306, 1997.

[7] D. J. Frank, Y. Taur, M. Ieong, and H.-S. P. Wong, "Monte Carlo modeling of threshold variation due to dopant fluctuations," in 1999 Symp. VLSI Technology Dig. Tech. Papers, 1999, pp. 169-170.
[8] A. Asenov, "Random dopant induced threshold voltage lowering and fluctuations in sub $0.1 \mu \mathrm{m}$ MOSFETs: A 3D 'atomistic' simulation study," IEEE Trans. Electron Devices, vol. 45, pp. 2505-2513, 1998.

[9] F. Stern, "Self-consistent results for n-type Si inversion layers," Phys. Rev. B, vol. 5, pp. 4891-4899, 1972.

[10] T. Ando, A. B. Fowler, and F. Stern, "Electronic properties of two-dimensional systems," Rev. Mod. Phys., vol. 54, pp. 437-672, 1982.

[11] S. Jallepalli, J. Bude, W.-K. Shih, M. R. Pinto, C. M. Maziar, and A. F. Tasch Jr., ""Electron and hole quantization and their impact on deep submicron silicon p- and n-MOSFET characteristics," IEEE Trans. Electron Devices, vol. 44, pp. 297-303, 1997.

[12] Y. Ohkura, "Quantum effects in Si n-MOS inversion layer at high substrate concentrations," Solid-State Electron., vol. 33, pp. 1581-1585, 1990.

[13] M. J. Van Dort, P. H. Woerlee, A. J. Walker, C. A. H. Juffermans, and H. Lifka, "Influence of high substrate doping levels on the threshold voltage and the mobility of deep submicron MOSFET's," IEEE Trans. Electron Devices, vol. 39, p. 932, 1992.

[14] G. Chindalore, S. A. Hareland, S. Jallepalli, A. F. Tasch Jr., C. M. Maziar, V. K. F. Chia, and S. Smith, "Experimental determination of threshold voltage shifts due to quantum mechanical effects in MOS electron and hole inversion layer," IEEE Electron Device Lett., vol. 18, pp. 206-208, 1997.

[15] A. S. Spinelli, A. Benvenuti, and A. Pacelli, "Self consistent 2-D model for quantum effects in n-MOS transistors," IEEE Trans. Electron Devices, vol. 45, pp. 1342-1349, 1992.

[16] A. Asenov, A. R. Brown, J. H. Davies, and S. Saini, "Hierarchical approach to 'atomistic' 3-D MOSFET simulation," IEEE Trans. Computer-Aided Design, vol. 18, pp. 1558-1565, 1999.

[17] K. Nishiohara, N. Shiguo, and T. Wada, "Effects of mesoscopic fluctuations in dopant distributions on MOSFET threshold voltage," IEEE Trans. Electron Devices, vol. 39, pp. 634-639, 1992.

[18] V. K. De, X. Tang, and D. J. Meindl, "Random MOSFET parameter fluctuation limits to gigascale integration (GSI)," in Tech. Dig., VLSI Symp.' 96, pp. 198-199.

[19] P. A. Stolk, F. P. Widdershoven, and D. B. M. Klaassen, "Modeling statistical dopant fluctuations in MOS transistors," IEEE Trans. Electron Devices, vol. 45, pp. 1960-1971, 1998.

[20] D. Vasileska, W. J. Gross, and D. K. Ferry, "Modeling of deep-submicrometer MOSFETs: Random impurity effects, threshold voltage shifts and gate capacitance attenuation," in Ext. Abstr, IWEC-6, Osaka, 1998, IEEE Cat. no. 98EX116, pp. 259-262.

[21] A. Asenov and S. Saini, "Suppression of random dopant induced threshold voltage fluctuations in sub- $0.1 \mu \mathrm{m}$ MOSFET's with epitaxial and $\delta$-doped channels," IEEE Trans. Electron Devices, vol. 46, pp. 1718-1723, 1999.

[22] J.-R. Zhou and D. K. Ferry, "Three-dimensional simulation of the effect of random dopant distribution on conductance for deep submicron devices," in Proc. 3rd Int. Workshop Computational Electronics. New York: Plenum, 1994, pp. 74-77.

[23] C. S. Rafferty, B. Biegel, Z. Yu, M. G. Ancona, J. Bude, and R. W. Dutton, "Multidimensional quantum effects simulation using a densitygradient model and script-level programming technique," in Simulation of Semiconductor Processes and Devices, K. De Meyer and S. Biesemans, Eds. Berlin, Germany: Springer, 1998, pp. 137-140.

[24] M. G. Ancona, "Macroscopic description of quantum-mechanical tunneling," Phys. Rev. B, vol. 42, pp. 1222-1223, 1990.

[25] A. G. Ancona, Z. Yu, R. W. Dutton, P. J. Vande Vorde, M. Cao, and D. Vook, "Density-gradient analysis of tunneling in MOS structures with ultra-thin oxides," in Proc. SISPAD '99, pp. 235-238.

[26] E. Madelung, "The hydrodynamical picture of quantum theory," Z. Phys. , vol. 40, pp. 332-326, 1926.

[27] D. Bohm, "A suggested interpretation of the quantum theory in terms of hidden variables," Phys. Rev., vol. 85, pp. 166-193, 1952.

[28] J.-R. Zhou and D. K. Ferry, "Simulation of ultra-small GaAs MESFET using quantum moment equations," IEEE Trans. Electron Devices, vol. 39, pp. 473-478, 1992.

[29] M. G. Ancona and G. I. Iafrate, "Quantum correction to the equation of state of an electron gas in a semiconductor," Phys. Rev. B, Condens. Matter, vol. 39, pp. 9536-9540, 1989.

[30] J. A. Greenfield and R. W. Dutton, "Nonplanar VLSI device analysis using the solution of Poisson's equation," IEEE Trans. Electron Devices, vol. ED-27, pp. 1520-1532, 1980.

[31] A. Asenov and S. Saini, "Polysilicon gate enhancement of the random dopant induced threshold voltage fluctuations in sub-100-nm MOSFET's with ultrathin gate oxides," IEEE Trans. Electron Devices , vol. 47, pp. 805-812, Apr. 1999. 
[32] J. A. López-Villanueva, P. Cartujo-Casinello, J. Bankueri, F. Gamiz, and S. Rodriguez, "Effects of inversion layer centroid on MOSFET behavior," IEEE Trans. Electron Devices, vol. 44, pp. 1915-1922, 1999.

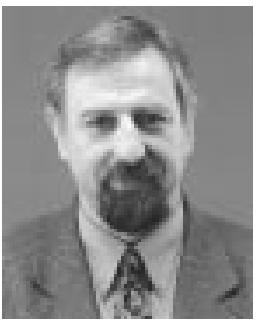

Asen Asenov (M'96) received the M.Sc. degree in solid-state physics from Sofia University, Bulgaria, in 1979, and the Ph.D. degree in physics from The Bulgarian Academy of Science, Sofia, in 1989.

He had ten years of industrial experience as a Head of the Process and Device Modeling Group in IME Sofia, developing one of the first integrated process and device CMOS simulators IMPEDANCE. From 1989 to 1991, he was a Visiting Professor at the Physics Department of TU Munich. He is currently Head of the Department of Electronics and Electrical Engineering at the University of Glasgow, Glasgow, U.K. As a leader of the Device Modeling Group and Academic Director of the Process and Device Simulation Center, he also coordinates the development of 2-D and 3-D device simulators and their application in the design of FETs, SiGe MOSFETs, and IGBTs. He has over 130 publications in process and device modeling and simulation, semiconductor device physics, "atomistic" effects in ultrasmall devices, and parallel computing.

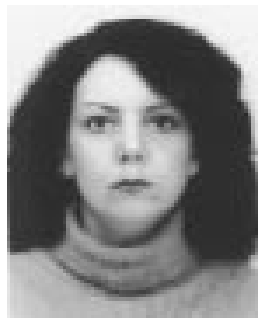

Gabriela Slavcheva received the Ph.D. degree in physics from The Bulgarian Academy of Sciences (BAS), Sofia, in 1997 for theoretical work on the electron and phonon spectra and transport phenomena in low-dimensional disordered systems. She is currently a Postdoctoral Fellow at the University of Glasgow, Glasgow, U.K.

She was a Research Associate at the Institute of Biophysics and Institute of Solid State Physics, BAS, Sofia. She spent two periods as a Visiting Professor at the Physics Department, Forum for Theoretical Science, Chulalongkorn University, Bangkok, Thailand, working on path-integral calculation of electronic properties at the interface of disordered heterostructures and as a Research Fellow in the Institute of Acoustics, Rome, Italy, working on the development of a 2-D solver for calculation of the acoustic phonon modes in composite membranes. Her research interests include theoretical investigation and modeling of electronic and optical properties of low-dimensional disordered systems with special attention to the effect of random impurity distribution on the ultrasmall device characteristics.

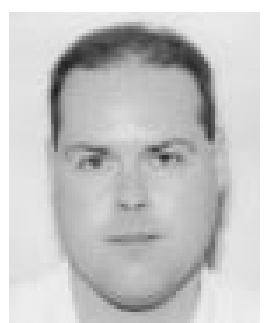

Andrew Brown received the B.Eng. degree in electronics and electrical engineering from the University of Glasgow, Glasgow, U.K., in 1992.

$\mathrm{He}$ is currently a Researcher in the Electrical Engineering Department at the University of Glasgow, working on the development of parallel 3-D simulators for semiconductor devices. He is currently developing a parallel 3-D "atomistic" simulator to investigate random dopant induced parameter fluctuations in sub-100 nm MOSFETs. Previous work include the simulation of IGBTs. His interests include high-performance parallel computing, device modeling, and visualization.

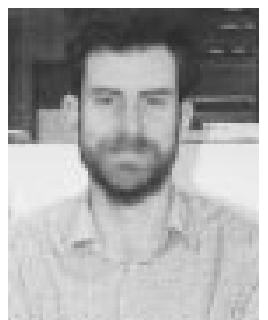

John H. Davies received the Ph.D. degree from the University of Cambridge, Cambridge, U.K., in 1982 for theoretical work on the electronic properties of amorphous semiconductors. He was a Research Fellow at Cornell University, Ithaca, New York.

In 1986, he joined Glasgow University, Glasgow, U.K., and has since spent two periods of leave at Ohio State University, Columbus, and the University of California at Santa Barbara. Most of his research is centered on the physics of transport in III-V heterostructures. This has included the modeling of surfaces and gates, including the effect of stress from patterned surfaces and gates. He has been interested in the effect of discrete, random donors for many years, and previous research showed their destructive effect on quantum transport in ballistic devices at low temperature. Other interests include the theory of resonant tunneling, conduction in lateral superlattices, and the calculation of magnetic fields in permanent-magnet motors.

Subhash Saini received the Ph.D. degree from the University of Southern California, Los Angeles.

He has held positions at the University of California at Los Angeles (UCLA), the University of California at Berkeley (UCB), and Lawrence Livermore National Laboratory (LLNL) (LOCATION?). He has over ten years of experience teaching physics at the B.S. (Hons) and M.S. levels. His research interests involve performance evaluation and modeling of a new generation of CMOSbased processors and highly parallel computers. He has published over 80 technical papers in nuclear engineering, quantum scattering, nanotechnology, hightemperature materials, operating systems, computer architectures, and performance modeling of high-end computers, and has presented over 100 technical talks. He joined NAS in 1989 and he was named the NAS-NASA employee of the year in 1991. Currently, he is Manager of a department at NASA Ames Research Center, Moffett Field, CA, which includes several groups such as Information Systems Performance Modeling; Algorithms, Architectures and Application; Information Power Grid Architectures; Legacy Codes Modernization; Higher Level Languages; Nanotechnology; and Device Modeling. 